\title{
Low lumbar CSF levels of homovanillic acid and 5-hydroxyindoleacetic acid in multiple system atrophy with autonomic failure
}

\author{
R J POLINSKY,* R T BROWN, * R S BURNS, $\dagger$ J HARVEY-WHITE, $\ddagger$ I J KOPIN \\ From the Clinical Neuropharmacology Section, ${ }^{*}$ and the Section on Aminergic Mechanisms, $\ddagger$ Clinical \\ Neuroscience Branch, NINCDS, Bethesda, MD, and the Department of Neurology, $\dagger$ Vanderbilt University \\ School of Medicine, Nashville, Tennessee, USA
}

SUMMARY Low lumbar CSF concentrations of homovanillic acid (HVA) and 5-hydroxyindoleacetic acid (5-HIAA) in patients with multiple system atrophy attended by autonomic failure (MSA) reflect decreased activity in central dopaminergic and serotonergic pathways. These neurochemical changes are consistent with the neuropathological involvement in MSA and distinguish such patients from those with pure autonomic failure who have normal CSF metabolite levels.

Pure autonomic failure (PAF) is a syndrome of chronic autonomic dysfunction that occurs in the absence of peripheral neuropathy or signs of a central neurological disturbance. ${ }^{1}$ Biochemical and pharmacological evidence supports primary involvement of the peripheral autonomic nervous system in the pathophysiology of the disorder but its underlying aetiology remains to be identified. ${ }^{2}$ Multiple system atrophy (MSA) encompasses the overlapping syndromes of olivopontocerebellar atrophy (OPCA) and striatonigral degeneration; ${ }^{3}$ in many patients MSA is attended by autonomic failure that appears to be primarily caused by central nervous system lesions. ${ }^{4}$

Monoamine neurotransmitters play an important role in the function of central and peripheral neuronal pathways. Measurement of plasma and urinary levels of neurotransmitters and their metabolites provides an index of peripheral function and metabolism. ${ }^{5}$ The relationship between norepinephrine and peripheral sympathetic nervous system activity has been extensively studied using this approach. ${ }^{6}$ Assessment of central nervous system neurotransmitter metabolism is limited to relatively indirect methods although the metabolite levels in CSF have been correlated with a variety of behavioural and functional characteristics. ${ }^{5,7}$ Previously, we showed that CSF levels

Address for reprint requests: Ronald J Polinsky, MD, National Institutes of Health, Building 10, Room 5N236, 9000 Rockville Pike, Bethesda, MD 20892, USA.

Received 1 December 1987 and in revised form 11 March 1988. Accepted 15 March 1988 of 3-methoxy-4-hydroxyphenylglycol (MHPG), corrected for the plasma contribution, are low only in patients with MSA attended by autonomic failure. ${ }^{8}$ This result demonstrated dysfunction in central noradrenergic neurons consistent with post-mortem histological and neurochemical findings in MSA. Dopamine and serotonin are neurotransmitters in the extrapyramidal system as well as the central pathways concerned with blood pressure regulation. There have been few clinical investigations of these neurotransmitters in a small number of patients with MSA. ${ }^{9-11}$ To our knowledge there have been no studies of patients with PAF. Since the clinical manifestations of autonomic dysfunction in PAF are similar to those in MSA, measurement of CSF metabolites in patients with PAF will reveal whether autonomic failure per se causes changes in central dopamine and serotonin function. Furthermore, the observation of neuropathological changes in the substantia nigra of a patient without central neurological signs who died 4 years after the onset of autonomic failure forms the basis for the suggestion that PAF might be a "form fruste" of Parkinson's disease. $^{12}$ Nigrostriatal dopaminergic involvement in PAF would be expected to produce a reduction in CSF levels of homovanillic acid as occurs in Parkinson's disease. ${ }^{9,10}$ To investigate the central dopaminergic and serotonergic systems, we have measured CSF levels of homovanillic acid (HVA) and 5-hydroxyindoleacetic acid (5-HIAA) in autonomic failure patients with either PAF or MSA and in normal subjects. 


\section{Materials and methods}

All patients had autonomic failure as a result of either PAF or MSA. The age range of the PAF group (four men, 10 women) was 36 to 75 (mean, SEM $=59.9,2 \cdot 9$ ) years. The MSA group is comprised of two overlapping clinical syndromes. Those patients with the striatonigral degeneration variant had pyramidal tract signs, rigidity out of proportion to bradykinesia and tremor, and evidence of diffuse central nervous system involvement. Patients with cerebellar dysfunction (OPCA) also manifested pyramidal and diffuse neurological signs in addition to the autonomic disorder. Some MSA patients had features common to both striatonigral degeneration and OPCA variants. For the purposes of correlating neurochemical measurements with clinical status, the patients with MSA were subdivided according to the presence and severity of Parkinsonian features (table); their mean (SEM) age was $54.3(1.4)$ years. In addition, extrapyramidal features in the MSA group were quantified by an independent observer using a modified Columbia scale with a maximum rating of $60 .{ }^{13}$ Control subjects were free of any medical illness and did not take any routine medications; they were recruited through the NIH normal volunteer programme. The normal volunteer group consisted of 14 men and 10 women who ranged in age from 23 to 71 (mean, $\mathrm{SEM}=46.3,3.0$ ) years. Informed consent was obtained in accordance with NIH guidelines. Each subject underwent a complete medical evaluation which included a neurological examination, screening blood studies, and electrocardiogram before lumbar puncture. The patients' medications were stopped for at least 1 week before the study. Diet was unrestricted during the hospital stay.

For lumbar puncture, subjects remained supine and fasted, except for water, from midnight until $0600 \mathrm{~h}$ when they were awakened and requested to urinate. They then remained flat in bed until the procedure was started at approximately $0800 \mathrm{~h}$. All lumbar punctures were completed before $0900 \mathrm{~h}$. Lumbar puncture was performed in either the sitting or lateral decubitus position. Following local cleansing with iodine and alcohol, xylocaine $(1 \%)$ was injected subcutaneously. An 18 or $20 \mathrm{G}$ spinal needle was inserted into either the L3-4 or L2-3 interspace. Opening pressure and visual appearance of the CSF was normal in all subjects. Routine CSF studies were obtained from the initial $4 \mathrm{ml}$ of CSF. Additional CSF $(23 \mathrm{ml})$ was collected into a series of tubes that contained preservatives appropriate for each chemical analysis. All aliquots of CSF were immediately frozen on dry ice and stored at $-70^{\circ} \mathrm{C}$ until the time of assay.

Assay of CSF HVA and 5-HIAA. CSF levels of HVA and 5-HIAA were measured by a modification of a previously published gas chromatographic, mass spectrometric method. The tubes containing the $6^{\text {th }}$ to the $8^{\text {th }} \mathrm{ml}$ of CSF were used for analysis. ${ }^{14}$ This particular aliquot of lumbar CSF was chosen for the study as part of a standardised protocol for collecting CSF at the NIH. Use of the same aliquot for all subjects minimises the effects of CSF concentration gradients known to occur for monoamine neurotransmitter metabolites. ${ }^{5}$ Internal standards $25 \mathrm{ng},{ }^{2} \mathrm{H}_{5}$. HVA and $100 \mathrm{ng},{ }^{2} \mathrm{H}_{2}$. 5-HIAA dissolved in $1 \mathrm{ml}$ of $0.2 \%$ ascorbic acid were added to 1-2 $\mathrm{ml}$ of CSF and the mixture acidified with $0.3 \mathrm{ml}$ of 3 $\mathrm{M} \mathrm{HCl}$. After addition of $50 \mu \mathrm{l}$ of $2.0 \%$ EDTA, the acidic metabolites were extracted into $5 \mathrm{ml}$ ethyl acetate. The organic phase was removed, dried with anhydrous $\mathrm{Na}_{2} \mathrm{SO}_{4}$ and evaporated under nitrogen. The residue was dissolved in $150 \mu \mathrm{l}$ of trifluoroethanol trifluoroacetic acid anhydride (1:2) and heated at $70^{\circ} \mathrm{C}$ for 15 minutes. After evaporation in a stream of nitrogen, the residue was dissolved in $200 \mu \mathrm{l}$ of trifluoroacetic acid anhydride and heated at $70^{\circ} \mathrm{C}$ for 60 minutes. After evaporating to dryness, the residue from this second evaporation was taken up into $50 \mu \mathrm{l}$ of dried ethyl acetate and the solution analysed by gas chromatographymass spectrometry (Finnigan 3200). Separation was achieved using a $1.5 \mathrm{~m} \times 2 \mathrm{~mm}$ (ID) glass column packed with $3 \%$ OV 17 on 100-120 mesh Gas Chrom Q (Applied Science, State College, PA). Column temperature was maintained at $155^{\circ} \mathrm{C}$ and $180^{\circ} \mathrm{C}$ for HVA and 5-HIAA respectively. Ion pairs were monitored at mass-to-charge ratio $(\mathrm{m} / \mathrm{z}) 360$ and 365 for endogenous and deuterated HVA and either 465 and 467 or 338 and 340 for endogenous and deuterated 5-HIAA respectively. Peak height ratios, after correction for channel crossover and natural abundance of deuterium, were used for determining the amounts of endogenous HVA and 5-HIAA by comparison with standard curves.

Statistical analysis of results. Mean (SEM) levels of CSF HVA and 5-HIAA were calculated for each group, and statistical differences were determined by analysis of variance with appropriate follow-up tests. The relationship between the metabolites was examined by linear regression.

Table Clinical characteristics of patients with multiple system atrophy

\begin{tabular}{|c|c|c|c|c|}
\hline Number & $\begin{array}{l}\text { Age } \\
(y r)\end{array}$ & Sex & $\begin{array}{l}\text { CSF } H V A \\
(n g / m l)\end{array}$ & $\begin{array}{l}\text { Clinical } \\
\text { rating }\end{array}$ \\
\hline $\begin{array}{r}1 \\
2 \\
3 \\
4 \\
5 \\
6 \\
7 \\
8 \\
9 \\
10 \\
11 \\
12 \\
13 \\
14 \\
15 \\
16 \\
17 \\
18 \\
19 \\
20 \\
21 \\
22 \\
23 \\
24 \\
25 \\
26 \\
27 \\
28 \\
29 \\
30 \\
31 \\
32 \\
33\end{array}$ & $\begin{array}{l}49 \\
55 \\
64 \\
50 \\
59 \\
38 \\
56 \\
67 \\
56 \\
59 \\
53 \\
62 \\
60 \\
40 \\
53 \\
57 \\
53 \\
53 \\
50 \\
55 \\
68 \\
54 \\
59 \\
33 \\
61 \\
63 \\
62 \\
40 \\
54 \\
61 \\
42 \\
51 \\
44\end{array}$ & 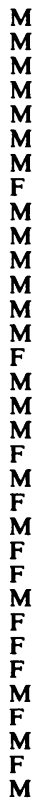 & $\begin{array}{r}21 \cdot 30 \\
24 \cdot 00 \\
20 \cdot 90 \\
9 \cdot 33 \\
18 \cdot 96 \\
17 \cdot 10 \\
14 \cdot 80 \\
39 \cdot 00 \\
16 \cdot 10 \\
15 \cdot 20 \\
31 \cdot 80 \\
11 \cdot 90 \\
31 \cdot 80 \\
15 \cdot 60 \\
24 \cdot 90 \\
19 \cdot 31 \\
6 \cdot 09 \\
5 \cdot 24 \\
7 \cdot 30 \\
23 \cdot 80 \\
35 \cdot 20 \\
5 \cdot 75 \\
13 \cdot 14 \\
7 \cdot 57 \\
10 \cdot 50 \\
17 \cdot 95 \\
40 \cdot 80 \\
12 \cdot 60 \\
16 \cdot 10 \\
19 \cdot 59 \\
8 \cdot 03 \\
19 \cdot 80 \\
5 \cdot 36\end{array}$ & $\begin{array}{r}0 \\
4 \\
4 \\
4 \\
5 \\
5 \\
6 \\
8 \\
9 \\
15 \\
16 \\
18 \\
20 \\
20 \\
23 \\
25 \\
25 \\
27 \\
28 \\
29 \\
30 \\
30 \\
32 \\
32 \\
34 \\
36 \\
39 \\
39 \\
41 \\
48 \\
48 \\
50 \\
59\end{array}$ \\
\hline
\end{tabular}




\section{Results}

The CSF levels of HVA and 5-HIAA in MSA (17.8, SEM 1.7 and 9.7 , SEM $0.7 \mathrm{ng} / \mathrm{ml}$ respectively) were significantly lower $(\mathrm{p}<0.0001)$ than in control subjects $(42.9,2.4$ and $18.2,0.9 \mathrm{ng} / \mathrm{ml}$ respectively) or patients with PAF. In PAF, the HVA and 5-HIAA levels in CSF $(38 \cdot 9,5 \cdot 1$ and $18 \cdot 1,1.7 \mathrm{ng} / \mathrm{ml}$ respectively) were not significantly different from those in the normals (fig 1). The mean age of the control subjects was significantly lower than the patients with PAF $(p<0.01)$ or MSA $(p<0.05)$. However, neither age nor sex was related to the CSF levels of HVA or 5-HIAA in any group. There was a significant correlation between HVA and 5-HIAA in normal subjects (fig $2, r=0.669, p<0.001$ ); a similar relationship was observed in patients with either PAF or MSA.

Although there was a tendency for MSA patients with higher Parkinsonian ratings to have lower CSF HVA levels, there was not a significant correlation between the quantitative clinical rating and the CSF metabolite level. However, patients with MSA were divided into three groups according to the overall

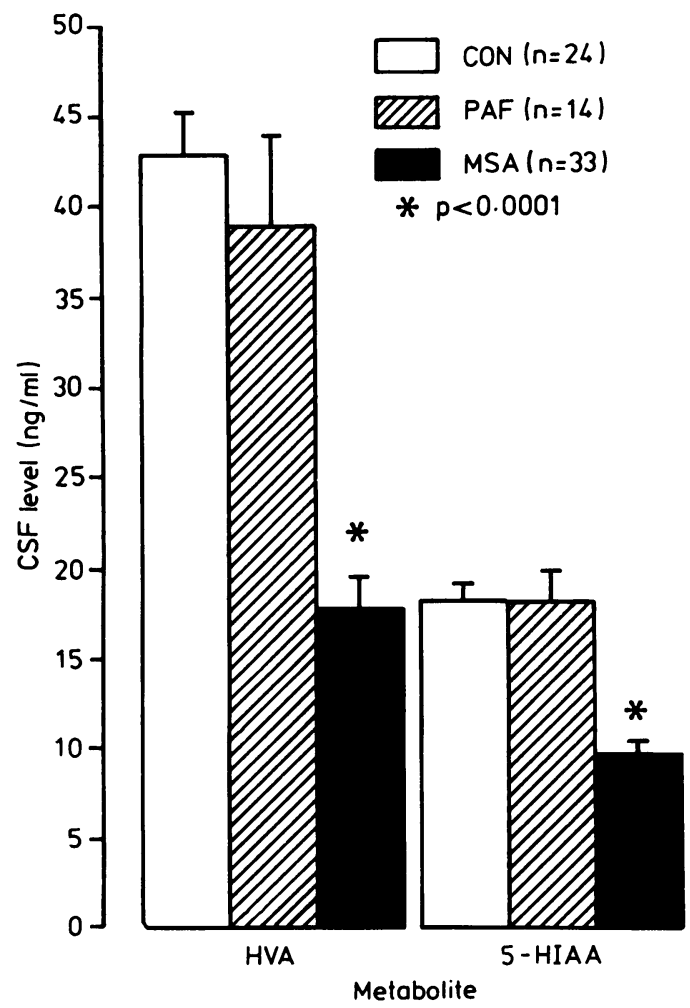

Fig 1 CSF levels (mean, SEM) of HVA and 5-HIAA in control subjects and patients with PAF or MSA.

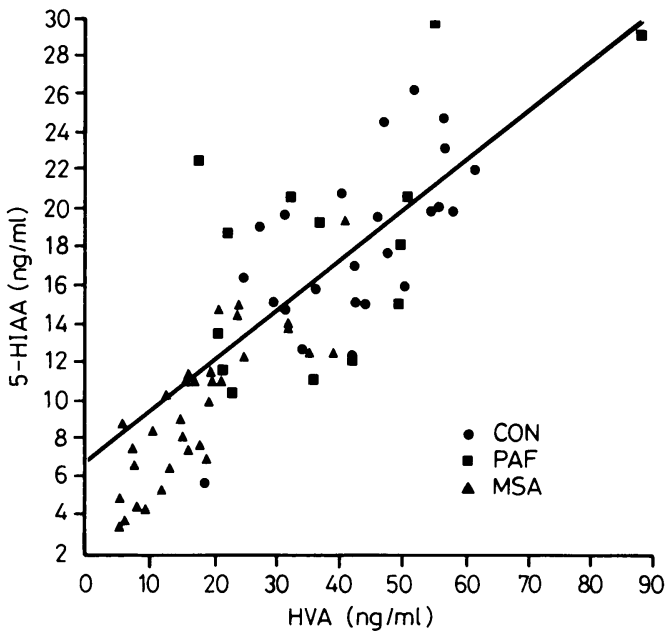

Fig 2 The relationship between CSF HVA and 5-HIAA. The regression line for normal subjects is shown.

clinical assessment of Parkinsonian features as absent $(N=6)$, present $(N=19)$, or severe $(N=8)$. Those patients with absent and severe Parkinsonism had quantitative ratings according to the modified Columbia scale that were significantly lower $(4 \cdot 3,1 \cdot 2)$ and higher $(38.8,3.9)$ respectively than the group with mild to moderate clinical features $(24 \cdot 9,2 \cdot 9)$ (fig 3$)$. All subgroups of MSA patients had CSF HVA and 5-HIAA levels which were lower than in normal controls. However, only those MSA patients with severe Parkinsonism had CSF HVA levels that were significantly lower $(p<0.01)$ than the other patients. The CSF 5-HIAA levels were not different among the three subgroups of patients with MSA. Following treatment with Sinemet ${ }^{(\mathbf{R})}$ (75/750 mg for 3 days) in 2 MSA patients the HVA levels in CSF were significantly increased above baseline from 5.75 and $17.95 \mathrm{ng} / \mathrm{ml}$ to 25.12 and $133 \mathrm{ng} / \mathrm{ml}$ respectively. There was no change in the CSF level of 5-HIAA following administration of Sinemet ${ }^{(\mathbf{R})}$.

\section{Discussion}

The results of this study confirm and extend the findings reported previously in smaller groups of patients with MSA. ${ }^{9-11}$ Involvement of brain dopaminergic pathways in MSA was suggested in the early descriptions of the syndrome because of the clinical findings of Parkinsonism. ${ }^{15}$ Reduced levels of dopamine and HVA in specific brain regions were observed in Parkinson's disease ${ }^{16,17}$ and led to the development of various therapeutic strategies aimed at altering the balance between dopaminergic and cholinergic neurotransmitter activity in the extra- 


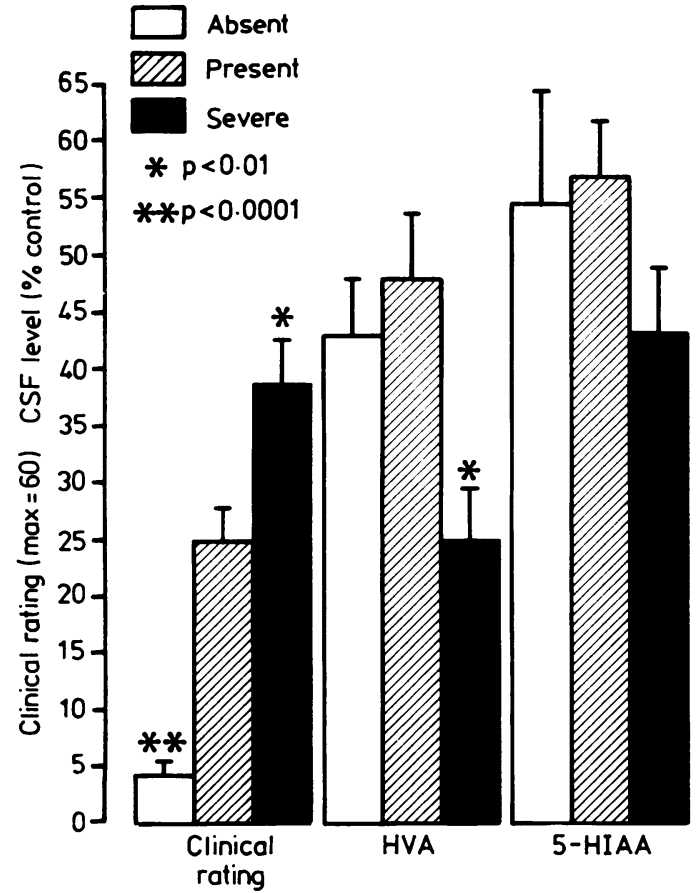

Fig 3 Clinical rating and CSF levels (expressed as percent of controls) of HVA and 5-HIAA in patients with MSA grouped according to the presence and severity of Parkinsonian features.

pyramidal system. Similarly, low CSF levels of HVA have been reported in patients with $\mathrm{MSA}^{9-11}$ and anti-Parkinsonian medications do improve the rigidity, bradykinesia, and tremor in the early stages of the disorder. ${ }^{18}$ The low levels of CSF HVA observed in our patients with MSA are similar to the significant reductions reported in Parkinson's disease patients. ${ }^{9-11,14}$ Neuronal loss ${ }^{19}$ and reduced dopamine content ${ }^{12}$ in the striatum and substantia nigra provide pathological and neurochemical evidence for the pathophysiological basis of Parkinsonism in MSA.

Decreased central serotonin turnover in MSA is suggested by the low levels of CSF 5-HIAA. Levels of this neurotransmitter metabolite in CSF also tend to be lower than normal in patients with Parkinson's disease $^{9.10 .14}$ but significant reductions have been infrequently observed. ${ }^{7.11}$ Serotonin is present in a number of brain regions affected in MSA; however, neurochemical studies in post-mortem brain samples have not consistently revealed significant reductions in serotonin to confirm dysfunction in this neurotransmitter system. ${ }^{12}$ Serotonergic involvement in MSA would not be surprising since the serotonin content is reduced in the substantia nigra, caudate, and putamen in Parkinson's disease. ${ }^{20}$ The origins of lumbar CSF HVA and 5-HIAA must also be considered in the interpretation of our findings. There is little doubt that CSF levels of HVA reflect brain dopamine metabolism (for review, see reference 5). In contrast, the relative contributions from brain and spinal cord to CSF 5-HIAA levels are less clear. A spinal cord contribution has been suggested on the basis of a rostral-caudal gradient for CSF 5-HIAA concentration which is much less than for HVA. ${ }^{21}$ However, patients with spinal cord blockage had CSF 5-HIAA levels that were similar to those without a block. ${ }^{22}$ Independent estimates by two groups agree that approximately one-third of the CSF 5-HIAA level results from spinal cord serotonin metabolism. ${ }^{23,24}$ Thus, the significant reduction in lumbar CSF levels of 5-HIAA in patients with MSA probably is due at least in part to a diminished contribution from spinal cord serotonergic pathways. Although serotonergic involvement in the spinal cord has not been reported, this anatomical region is clearly the site of well-documented morphological changes in MSA. ${ }^{19}$

The CSF concentrations of HVA and 5-HIAA apparently increase with age, ${ }^{7}$ but we did not observe such a correlation. Since the mean age of the controls in this study was less than the patients. any expected age related increase would actually bias against the significantly reduced levels of monoamine metabolites observed in MSA. As expected, MSA patients with the most severe Parkinsonism had the lowest CSF levels of HVA supporting a pathophysiological relationship between brain dopamine and extrapyramidal motor function. However, even those MSA patients without Parkinsonian features, that is, olivopontocerebellar atrophy (OPCA), had significantly reduced HVA compared to normals. This is consistent with the widespread neuropathological changes in OPCA which may include the basal ganglia. The lack of clinical evidence for extrapyramidal dysfunction in the OPCA subgroup of MSA patients is probably related to the degree of involvement; it has been estimated that approximately $80 \%$ of neuronal function must be affected before symptoms appear in Parkinson's disease. ${ }^{20}$ In addition, there are other brain dopamine systems that are unrelated to motor control. ${ }^{25}$ These features as well as the imprecise nature of a clinical rating scale probably account for our inability to achieve a statistically significant correlation between the quantified assessment of Parkinsonism and CSF HVA levels in MSA, even among only those patients with Parkinsonian features. The similar reductions of 5-HIAA among the three subgroups of MSA patients divided according to severity of Parkinsonism suggests that the serotonergic dysfunction in MSA may not be 
primarily related to the motor deficit. The central nervous system distribution of serotonin differs from dopamine and it may play a more prominent role in relation to brainstem control of wakefulness, blood pressure and other vegetative functions. ${ }^{26}$

The intercept of the linear regression of 5-HIAA on HVA levels in CSF indicates that a portion of 5-HIAA is derived from a source which is independent of HVA. This is not surprising since clearly not all serotonergic neuronal activity is linked to that of dopaminergic neurons. It is perhaps more surprising that among the normal subjects as well as among the whole group of patients including those MSA patients with much diminished CSF levels of HVA and 5-HIAA there is a highly significant correlation between the two amine metabolites. The basis of this correlation is unclear, but several possible mechanisms might serve as the basis for this relationship. A physiological link between the two aminergic systems appears most likely. Since correction of the dopamine deficit by levodopa treatment in two patients did not alter the 5-HIAA level, it appears unlikely that this link is due to dopaminergic regulation of serotonergic function. The remaining possibilities for the normal subjects are that both aminergic systems are regulated by a third or several other systems or that the serotonergic system exerts a strong modulatory effect on the dopaminergic system. In the patients with MSA, this third system could be damaged, or the two aminergic systems similarly involved in the degenerative process. The elevation in HVA following Sinemet ${ }^{(\mathrm{R})}$ indicates that the capacity to increase dopamine turnover may be preserved in MSA and probably accounts for the improvement observed in response to anti-Parkinsonian drugs. Of interest is the finding reported by Williams et al that patients with MSA decrease their CSF levels of HVA in response to bromocriptine. ${ }^{10}$ Thus, it appears that some pre-synaptic mechanisms including dopamine synthesis, release, metabolism and receptor activation may remain functional in MSA.

The normal CSF levels of monoamine neurotransmitters in PAF are consistent with the results of other biochemical and pharmacological investigations that indicate peripheral autonomic involvement. ${ }^{4}$ Unfortunately, there has not been a complete, detailed post-mortem examination of the nervous system in PAF. Oppenheimer suggested a relationship between PAF and Parkinson's disease on the basis of depigmentation and Lewy bodies in the substantia nigra. ${ }^{19}$ Our data would not support that hypothesis since the HVA levels were quite normal and the patients have not developed any symptoms or signs of Parkinsonism while having PAF for as long as 25 years. Thus, the results of this study provide further biochemical evidence for distinguishing between PAF and MSA despite the clinical similarity in the manifestations of autonomic failure.

We acknowledge the assistance of the nursing staffs of Ward 3 East and Ward 5 East, NIH Clinical Center, who cared for the patients and assisted with the procedures in this study. Linda Nee, MSW, provided emotional support for the patients and recruited subjects for this investigation. Ms Ligia Ferreira assisted in manuscript preparation.

\section{References}

1 Bradbury S, Eggleston C. Postural hypotension: A report of three cases. Am Heart J 1925;1:73-86.

2 Polinsky RJ. Pharmacological responses and biochemical changes in autonomic failure. In: Bannister Sir R, ed. Autonomic Failure. London: Oxford University Press, 1983:201-36.

3 Bannister R, Oppenheimer DR. Degenerative diseases of the nervous system associated with autonomic failure. Brain 1972; 95:457-74.

4 Polinsky RJ, Kopin IJ, Ebert MH, Weise V. Pharmacologic distinction of different orthostatic hypotension syndromes. Neurology 1981;31:1-7.

5 Kopin IJ. Catecholamine metabolism: basic aspects and clinical significance. Pharmacol Rev 1985;37:333-64.

6 Goldstein DS, McCarty R, Polinsky RJ, Kopin IJ. Relationship between plasma norepinephrine and sympathetic neural activity. Hypertension 1983;5:552-9.

7 Scheinin M. Monoamine metabolites in human cerebrospinal fluid: indicators of neuronal activity? Med Biol 1985;63:1-7.

8 Polinsky RJ, Jimerson DC, Kopin IJ. Chronic autonomic failure: CSF and plasma 3-methoxy-4-hydroxyphenylglycol. Neurology 1984;34:979-83.

9 Curzon G. Involuntary movements other than Parkinsonism: biochemical aspects. Proc R Soc Med 1973;66:873-6.

10 Williams AC, Nutt J, Lake CR et al. Actions of bromocriptine in the Shy-Drager and Steele-Richardson-Olszewski syndromes. In: Fuxe K, Calne DB, eds. Dopaminergic Ergots and Motor Control. New York: Pergamon Press, 1979:271-83.

11 Ichikawa N. Study on monoamine metabolite contents of cerebrospinal fluid in patients with neurodegenerative diseases. Tohoku J Exp Med 1986;150:435-46.

12 Spokes EGS, Bannister R, Oppenheimer DR. Multiple system atrophy with autonomic failure: clinical, histological, and neurochemical observations on four cases. J Neurol Sci 1979; 43:59-82.

13 Duvoisin RC. The evaluation of extrapyramidal disease. In: de Ajuriaguerra J, Gauthier G, eds. Monoamines Noyaux Gris Centraux et Syndrome de Parkinson. Paris: Masson, 1971: 313-25.

14 Burns RS, Chiueh CC, Markey SP, Ebert MH, Jacobowitz DM, Kopin IJ. A primate model of Parkinsonism: Selective destruction of dopaminergic neurons in the pars compacta of the substantia nigra by $\mathrm{N}$-methyl-4-phenyl-1, 2, 3, 6-tetrahydropyridine. Proc Natl Acad Sci 1983;80:4546-50.

15 Shy GM, Drager GA. A neurological syndrome associated with orthostatic hypotension: A clinical-pathologic study. Arch Neurol 1960;2:511-27.

16 Hornykiewicz O. Parkinson's disease: from brain homogenate to treatment. Fed Proc 1973;32:183-90.

17 Hornykiewicz O. Brain dopamine in Parkinson's disease and other neurological disturbances. In: AS Horn, ed. The Neurobiology of Dopamine. New York: Academic Press, 1979: 633-54.

18 Polinsky RJ. Multiple system atrophy: Clinical aspects, patho- 
physiology, and treatment. Neurol Clin 1984;2:487-98.

19 Oppenheimer D. Neuropathology of progressive autonomic failure. In: Bannister Sir R, ed. Autonomic Failure. London: Oxford University Press, 1983:267-83.

20 Hornykiewicz $\mathrm{O}$. Imbalance of brain monoamines and clinical disorders. Prog Brain Res 1982;55:419-29.

21 Sjostrom R, Ekstedt J, Anggard E. Concentration gradients of monoamine metabolites in human cerebrospinal fluid. J Neurol Neurosurg Psychiatry 1975;38:666-8.

22 Post RM, Goodwin FK, Gordon E, Watkin DM. Amine metabolites in human cerebrospinal fluid: Effects of cord transection and spinal fluid block. Science 1973;179:897-9.
23 Weir RL, Chase TN, Ng LKY, Kopin IJ. 5-hydroxyindoleacetic acid in spinal fluid: Relative contribution from brain and spinal cord. Brain Res 1973;52:409-12.

24 Garelis E, Sourkes TL. Sites of origin in the central nervous system of monoamine metabolites measured in human cerebrospinal fluid. J Neurol Neurosurg Psychiatry 1973;36:625-9.

25 Dray A. The physiology and pharmacology of mammalian basal ganglia. Progr Neurobiol 1980;14:221-335.

26 Dahlstrom A, Fuxe K. Evidence for the existence of monoamine-containing neurones in the central nervous system: $I$. Demonstration of monoamines in the cell bodies of brain stem neurones. Acta Physiol Scand 1964;62(Suppl:232):1-55. 